\title{
Dynamiques de structuration des bassins laitiers : comparaison entre l'Amazonie brésilienne et le Mali
}

\author{
R. Poccard-Chapuis ${ }^{1,2 *}$ C. Corniaux ${ }^{2,3}$ D. Coulibaly ${ }^{3}$
}

\begin{abstract}
Mots-clés
Bovin laitier - Développement agricole - Modèle - Secteur informel Amazonie - Brésil - Mali.
\end{abstract}

\begin{abstract}
Résumé
En Amazonie brésilienne, des processus rapides de structuration de bassins laitiers sont en cours. Au Mali, cette structuration n'est pas enclenchée, bien que l'élevage bovin soit une activité ancienne et en pleine expansion. Cette différence recouvre des enjeux essentiels : lutte contre la pauvreté, productions alimentaires face au défi démographique, débats actuels sur les théories du développement. L'analyse comparative s'appuie sur une modélisation diachronique des phases successives de structuration des bassins laitiers amazoniens. Ce modèle est ensuite appliqué aux dynamiques laitières maliennes pour identifier les blocages dans la structuration des bassins. Le modèle amazonien montre six phases, depuis l'autoconsommation de lait cru jusqu'à la spécialisation du bassin et l'instauration d'un monopole industriel. L'application au Mali montre que les situations initiales sont similaires (contraintes liées au territoire). Mais deux mécanismes essentiels ne fonctionnent pas : le transport de produits finis vers des marchés distants et la collecte du lait frais dans un rayon supérieur à celui de la vente directe aux consommateurs urbains. Ces deux aspects, essentiels pour assurer la fluidité en amont et an aval de l'industrie, conduisent à revoir les lieux d'implantation des laiteries, ainsi que l'organisation de la distribution à Bamako. Malgré les limites de I'analyse comparative, le modèle montre que les clés d'une évolution positive au Mali se situent davantage au niveau des industries et de la distribution qu'au niveau de la production. Les actions publiques (Etat et collectivités) pourraient porter sur ces deux points, notamment par de la formation et des politiques de crédit.
\end{abstract}

\section{INTRODUCTION}

La commercialisation du lait est l'un des leviers privilégiés pour lutter contre la pauvreté dans les exploitations agricoles familiales des pays tropicaux (13). Dans ce cadre, la laiterie, opérateur de collecte et de transformation, représente un maillon essentiel du processus de développement. L'industrialisation laitière, que l'Europe a connue à partir du milieu du $\mathrm{XIX}^{\mathrm{e}}$ siècle, est actuellement

\footnotetext{
1. Embrapa Amazônia Oriental, TV Eneas Pinheiro, s/n Bairro Marco, Belém PA, CEP 66095-100, Brasil.

2. Cirad, UPR Systèmes d'élevage, Montpellier, F-34398 France.

3. Institut d'économie rurale, Centre régional de recherche agronomique de Sotuba, Bamako, Mali.

* Auteur pour la correspondance

Tél. : +559132041093; fax : +559132767939

E-mail : rene@cpatu.embrapa.br
}

en cours dans certaines régions du Sud : c'est le cas au Brésil. En revanche, la structuration de bassins laitiers autour des laiteries apparaît encore limitée dans d'autres régions, en dépit de leur tradition d'élevage : c'est le cas au Mali. Comment peut-on expliquer une telle différence?

Les enjeux de cette question sont de taille. Il s'agit en effet d'apporter à une population urbaine en croissance une alimentation protéique dans un contexte de faible solvabilité. Il s'agit également de réduire une dépendance vis-à-vis de la poudre de lait importée au profit de la production locale. Il s'agit enfin, de promouvoir un développement local, à partir d'une demande urbaine en produits agroalimentaires (le lait). D'un point de vue théorique, cette question est au cœur des débats fondamentaux sur la notion de développement.

Afin d'y apporter des éléments de réponse, l'article développe une comparaison entre deux cas, l'un au Brésil, l'autre au Mali. 
L'hypothèse des auteurs est que ce sont les mécanismes de structuration progressive des bassins laitiers qui déterminent la croissance de la production laitière, et non l'inverse. Ils constituent aussi un moteur qui accélère le développement local. Sur les fronts pionniers brésiliens, les territoires se structurent rapidement en bassins laitiers, dans une dynamique solide de développement local. Pour comprendre ce processus les auteurs ont élaboré un modèle diachronique, qui montre les phases successives de structuration des bassins laitiers. Ce modèle est ensuite appliqué au Mali afin d'identifier des blocages et de proposer des pistes d'action d'ordre technique, organisationnel et politique.

\section{- UN MODELE POUR COMPARER DES DYNAMIQUES DE BASSINS LAITIERS}

\section{Essor des filières laitières, au cour de l'économie du développement au Mali}

La production et la consommation de lait et produits laitiers sont très anciennes en Afrique de l'Ouest. On peut les qualifier de traditionnelles, notamment dans les sociétés sahariennes et sahéliennes (6). La culture peule est à cet égard très emblématique.

Depuis les années 1950, ces pays et en particulier le Mali connaissent un mouvement d'urbanisation qui s'est intensifié au cours des années 1990. Aujourd'hui, même si la population malienne reste rurale à 70 p. 100, Bamako compte près de 1,5 million d'habitants ; une demi-douzaine de villes secondaires dépasse les 50000 habitants. Dans ces pôles urbains, la consommation de lait augmente et de nouveaux modes de consommation des produits laitiers se vulgarisent. Actuellement, cette demande est majoritairement satisfaite par des importations de poudre (27). Les préférences des consommateurs urbains, tout comme l'intérêt économique et logistique des opérateurs de transformation et de distribution le long de la filière, portent sur le lait en poudre plutôt que sur le lait frais (21).

La production laitière locale se trouve ainsi confrontée à plusieurs contraintes pour accéder aux marchés urbains. Les unités de transformation ont besoin d'un approvisionnement régulier en qualité et en quantité qui n'est pas compatible avec les modes traditionnels de production laitière dans la région, fondés sur l'atomisation de l'apport laitier (chez les Peuls, le lait appartient en propre aux femmes), sa saisonnalité (la production laitière est concentrée dans la période d'hivernage, entre juin et septembre) et sur la mobilité des troupeaux. D'autre part, les circuits commerciaux mondialisés mettent en concurrence la production locale avec celle d'autres continents dans le cadre de normes et de règles commerciales fixées à l'échelle mondiale. La production locale est ainsi menacée sur ses propres marchés par les flux mondiaux de lait.

De ce point de vue, la filière laitière malienne illustre bien la problématique actuelle de l'économie du développement de ce pays : nouvelles demandes agroalimentaires, remise en cause brutale des modèles traditionnels de production et de distribution, concurrence non réglementée par des filières internationales plus compétitives. Derrière cette question abordée ici sous l'angle du développement local se trouve également une problématique nationale et stratégique. La souveraineté alimentaire du Mali serait mise en péril si la demande de lait n'était assurée que par des importations. Cela concerne en particulier les zones urbaines, dans la mesure où en zones rurales prédominent l'autoconsommation et le commerce local. L'épisode de hausses de prix sur le marché mondial observé en 2007 en est une illustration ; les émeutes urbaines observées dans les pays voisins en 2008 également (Bobo-Dioulasso et Dakar, février - mars 2008).
L'enjeu est donc de taille : il s'agit de savoir comment la demande urbaine de lait en croissance pourrait profiter aux filières locales. Le scénario négatif où les flux locaux sont totalement substitués par des importations est facile à imaginer car il est proche de la réalité actuelle. En revanche, les auteurs questionnent la plausibilité d'un scénario plus positif, où la demande urbaine pourrait devenir un facteur de développement des filières locales. Leur objectif est d'analyser les mécanismes qui, à l'échelle des bassins laitiers, pourraient amorcer puis faire monter en puissance un réel développement local autour des filières laitières.

\section{Principes d'une comparaison entre Amazonie et Mali}

Le lait est souvent présenté comme un produit local, typique, ancré au terroir. Les produits laitiers possèdent une dimension culturelle très affirmée, leur diversité étant étroitement liée aux territoires et aux sociétés qui les habitent. Outre la dimension culturelle, ce lien entre les produits laitiers et les territoires se traduit également par des contraintes pour le développement des filières laitières, dont on peut retenir pour le Mali : les températures élevées, le réseau de transport précaire, le réseau de distribution d'énergie incertain et peu étendu, la faible capitalisation des acteurs, la faible technicité le long de la filière, les demandes spécifiques des consommateurs (pas de fromages). Ces contraintes sont montrées du doigt par les analystes comme responsables des échecs du développement laitier (12).

Or, des difficultés similaires s'observent sur les fronts pionniers amazoniens, bien que le contexte économique et culturel soit très différent. En Amazonie, la production laitière parvient à générer, dans certaines conditions, de véritables dynamiques de développement local. Partant de ce constat, les auteurs ont souhaité comparer les dynamiques des deux régions, pour tenter d'identifier des pistes de solutions applicables au Mali. Une telle démarche comparative, si elle est classique en économie du développement, se révèle périlleuse dans la mesure où les nombreuses différences entre les deux zones empêchent une comparaison stricto sensu. Il ne s'agira donc pas d'affirmer que « ce qui a marché là devrait être appliqué ici », ni que des phénomènes similaires dans leurs effets ont nécessairement les mêmes causes. Les deux pays correspondent à des sociétés différentes : les dynamiques laitières, étroitement liées aux valeurs culturelles, aux systèmes économiques et aux pratiques sociales, ne sauraient donc être assimilables. Le modèle construit n'est donc pas centré sur ces aspects.

La comparaison est basée sur le fonctionnement des bassins laitiers dans des contextes territoriaux contraignants. De ce point de vue, les notions de distance au marché, de dispersion des producteurs, de précarités des infrastructures, de carences en investissements, d'exigences des marchés, de procédés de transformation peuvent être appliquées de façon homogène. Les règles de la géographie et de l'économie des filières peuvent s'appliquer des deux côtés de l'océan. La modélisation de cette étude amènera à discuter du modèle de von Thünen - une référence pour expliquer la localisation des productions laitières en fonction des deux contraintes majeures de coût du foncier et du transport (34).

\section{Démarche de modélisation des bassins laitiers}

Dans cet article, l'accent est mis sur la notion de bassin laitier. Elle semble pertinente pour appréhender les différents facteurs techniques, économiques, organisationnels et sociaux, déterminant le succès ou l'échec d'une dynamique laitière. Autrement dit, étudier la dimension territoriale du fonctionnement d'une filière laitière permet de mieux comprendre les mécanismes de son développement ou de son déclin. 
L'approche territoriale des auteurs est centrée sur l'espace dans lequel s'inscrit la filière laitière locale. Le bassin laitier est défini à partir de la métaphore hydraulique : un territoire structuré autour d'un pôle marchand, drainant les flux de lait ou produits laitiers. La filière y joue un rôle important, dans la mesure où elle relie les acteurs qui assurent ces flux. La filière laitière intervient ainsi dans l'organisation spatiale du bassin (occupation de la terre, réseaux de transport, flux de marchandises, localisation des activités productives et des populations...) et dans son fonctionnement économique et social (systèmes d'activités, emplois urbains et ruraux, sécurité alimentaire, valeur ajoutée produite et externalités ...). Ainsi, le bassin laitier est construit par une filière laitière qui s'y installe, puis s'y développe, et qu'elle modifie pour améliorer son propre fonctionnement. Cet espace est drainé par des flux de matière première, polarisés autour d'unités de transformation, voire directement par le marché consommateur. Le pôle de consommation peut ou non être localisé au sein du bassin laitier, en être voisin ou distant, en fonction de règles explicitées par von Thünen (34) sur lesquelles les auteurs reviendront. Le bassin laitier peut donc être plus ou moins spécialisé, suivant l'importance relative de la production laitière et des autres activités agricoles. Il peut également être plus ou moins structuré, entre une phase embryonnaire jusqu'à une phase plus aboutie des interactions filière - territoire.

Le développement des bassins laitiers en Amazonie relève de mécanismes complexes. La diversité des situations est grande, tous les bassins laitiers de cette vaste région ne se ressemblent pas et n'en sont pas au même stade d'une histoire qui serait commune et invariable. En ce sens, il n'existe pas de modèle systématique expliquant la genèse de ces territoires où grandit une filière laitière. Malgré cela, il est possible d'identifier des constantes et des processus génériques. Les auteurs ont simplifié la description de ces processus, de façon à dresser une grille de lecture de cette complexité. Cette démarche a conduit à formaliser plusieurs configurations étagées sur une échelle de développement de ce système spatial qu'est le bassin laitier. On peut ainsi expliciter les mécanismes de structuration qui font évoluer les bassins laitiers. Chacune de ces configurations est ici représentée par un schéma graphique. Quelques bassins amazoniens, peu nombreux, ont connu successivement toutes ces configurations. Il ne s'agit donc pas d'un modèle purement théorique, puisqu'il s'appuie sur des données d'observation. Il n'est pas non plus universel, dans la mesure où tous les bassins laitiers ne passent pas obligatoirement par toutes ces phases. En revanche, tous se trouvent actuellement dans l'une ou l'autre des configurations décrites ici. Cette démarche diachronique décrivant des phases de développement s'apparente à celle des « étapes de la croissances économique » définies par Rostow (29). Elle ne s'applique cependant pas à des économies nationales, mais à la structuration de territoires autour d'une filière.

En d'autres termes, en formalisant cette suite de configurations les auteurs ont dressé un modèle global de compréhension des dynamiques de bassins laitiers en Amazonie.

\section{DYNAMIQUES DE BASSINS LAITIERS AMAZONIENS}

\section{Amazonie orientale : des bassins laitiers émergent sur les fronts pionniers}

Les dynamiques agraires en Amazonie orientale brésilienne sont marquées par les fronts pionniers de déforestation. Localisés sur la périphérie du grand massif forestier amazonien, ceux-ci correspondent à des lieux de transformation de l'espace. De nouvelles régions d'élevage s'y structurent (25). Le milieu naturel forestier, habité et exploité par des sociétés indigènes, est transformé par les pionniers en espaces déforestés, dominés par les pâturages cultivés et l'élevage bovin extensif. A l'avant du front, la ligne de déforestation avance sur la forêt et laisse derrière elle un espace neuf qui progressivement se structure, jusqu'à l'intégration aux régions développées du pays. Des pistes sont percées et entretenues, le foncier est réparti, les circuits commerciaux s'installent, des exploitations se spécialisent dans le naissage ou l'embouche, les systèmes techniques se précisent, l'approvisionnement en intrants s'organise, des industries de transformation s'installent, des externalités positives se dégagent, une société locale se consolide autour des métiers et fonctions de l'élevage et sa filière (18). La petite région encore en gestation se spécialise dans l'élevage, sans qu'aucune autre filière agricole n'y ait encore trouvé des conditions suffisamment favorables pour s'implanter (à l'exception notoire de l'extraction et du sciage de quelques essences de bois précieux comme Swietenia macrophylla). Les fronts pionniers deviennent des pourvoyeurs de bovins bien conformés (plus de $240 \mathrm{~kg}$ équivalent carcasse à l'abattoir) et de lait cru possédant de bonnes caractéristiques physico-chimiques en toutes saisons.

Du point de vue de la filière, les vastes espaces forestiers amazoniens constituent une promesse d'extension des actuels bassins d'approvisionnement en lait et viande bovine. Dans un contexte mondial où le Brésil devient un exportateur de premier plan pour les productions animales, une telle promesse est une aubaine. S'implanter en Amazonie, c'est se donner les moyens de produire plus et de mieux profiter d'un marché toujours plus ouvert aux produits brésiliens, en l'occurrence amazoniens (24). La hausse du cours mondial du lait en 2007 a donné une nouvelle impulsion à cette dynamique de développement.

Les Brésiliens parviennent donc à surmonter les difficultés de fonctionnement des filières laitières et de structuration des bassins laitiers dans des territoires contraignants. Ils en font même un atout pour mieux prendre part aux échanges mondiaux et pour intégrer les périphéries économiques de leur immense territoire. Ceci est une caractéristique fondamentale de l'esprit pionnier qui prédomine sur les fronts amazoniens où " tout est à faire ». La dynamique des bassins laitiers amazoniens est liée à cette aspiration à entreprendre des pionniers.

\section{Modélisation des dynamiques amazoniennes}

La figure 1 est composée de six graphiques, représentant chaque configuration successive du bassin laitier amazonien, depuis la naissance du front pionnier jusqu'à la spécialisation du territoire et l'industrialisation laitière.

\section{Autoconsommation de lait cru}

Le terme d'autoconsommation est utilisé à l'échelle du bassin et non de la propriété rurale : le bassin consomme ce qu'il produit, sans échanges avec l'extérieur. La situation initiale est celle de l'enclavement. Aucun flux de produits n'est expédié hors du front pionnier ni même ne circule à l'intérieur du front. Tout se passe à l'échelle locale (communale). Chaque chef-lieu représente un tout petit marché pour le lait cru local, approvisionné en vente directe par les producteurs les plus proches (dans un rayon de quelques kilomètres). Il n'y a pas de transformation industrielle, ni artisanale à quelques exceptions près (certains éleveurs, plus éloignés et doués du savoir faire adéquat, transforment leur lait en fromage artisanal, vendu au chef-lieu les jours de marché). La ménagère urbaine stérilise elle même le lait en le faisant bouillir. Le système de vente directe garantit une bonne rémunération au producteur, c'est pourquoi il se maintient au cours du temps. La concurrence de produits extérieurs est de toute façon limitée, l'approvisionnement 

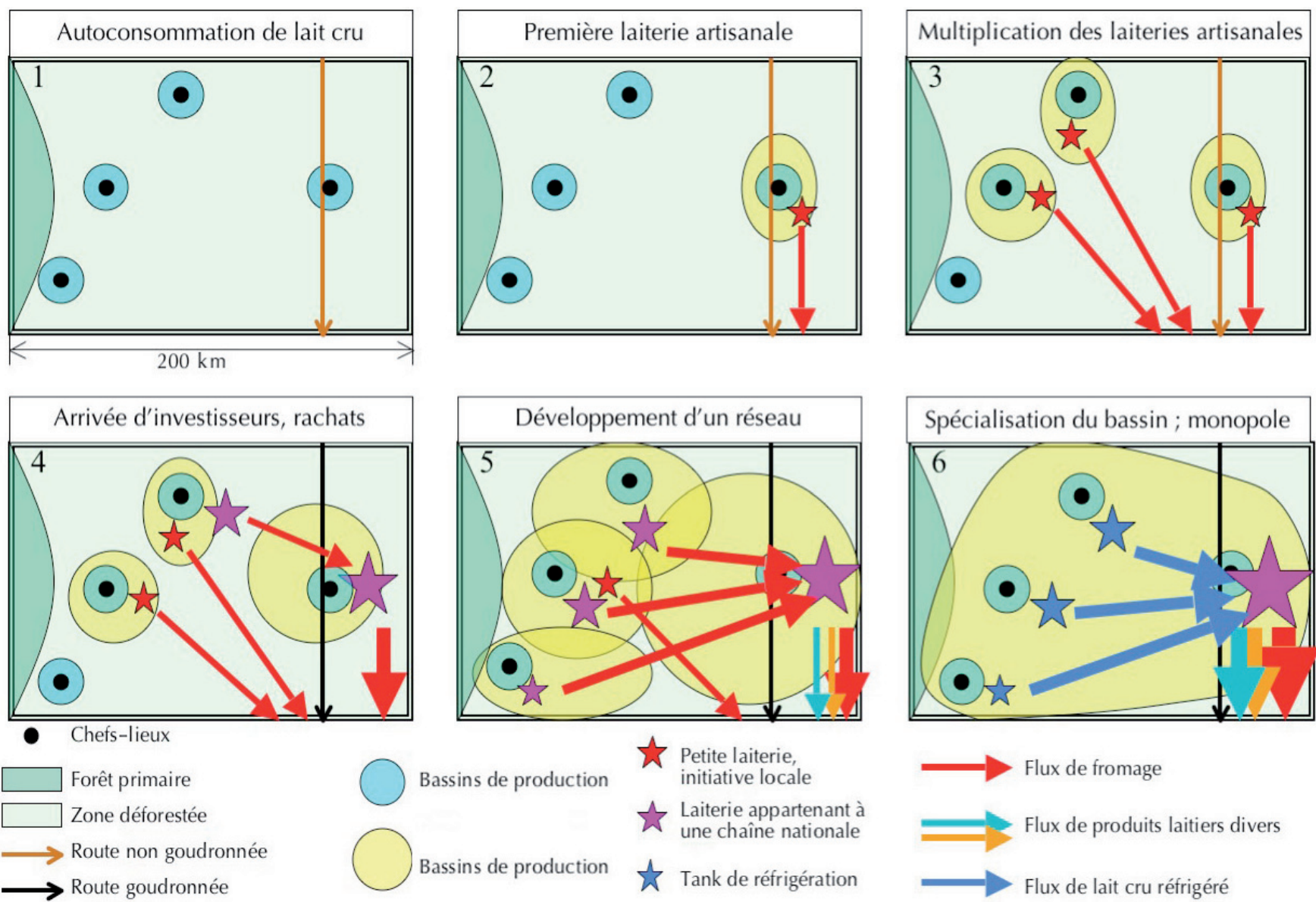

Figure 1 : évolution des bassins laitiers en Amazonie orientale brésilienne, succession de systèmes spatiaux structurants.

des petites villes pionnières étant réduit aux produits de première nécessité, à un prix élevé. Le lait en poudre est cependant présent, grâce en particulier à sa capacité de conservation.

Les bassins laitiers identifiables dans cette situation initiale sont de petite taille, le volume global commercialisé est de quelques centaines de litres par jour. Ils mobilisent très peu d'acteurs, de l'ordre d'une petite cinquantaine d'éleveurs. L'impact de la commercialisation du lait sur les dynamiques territoriales est faible ou nul. Cette configuration est cependant adaptée à une situation d'extrême précarité, typique des fronts pionniers les plus jeunes ou les plus enclavés. On peut parler d'embryon de bassin laitier.

\section{Premières laiteries artisanales}

Cette configuration peut se mettre en place autour des chefs-lieux importants, dont la population est de l'ordre de 10000 habitants. Le marché local est alors suffisant pour rentabiliser des équipements de base, d'autant plus que les habitants de ces villes n'ont pas, au contraire de beaucoup de Maliens, l'habitude de produire eux-mêmes leur lait en ville, ou de l'acheminer chaque jour depuis leur troupeau. D'autre part, ces premiers noyaux urbains abritent les acteurs disposant du minimum de capital nécessaire à l'établissement de laiteries artisanales. Ces conditions étant réunies, des procédés de transformation en yaourt et lait pasteurisé peuvent être mis en place. Un simple pasteurisateur, souvent artisanal, est suffisant. Ce genre d'établissement échappe aux services d'inspection et l'investissement requis est donc restreint (pas de normes sanitaires à respecter). Des entrepreneurs ou des associations peuvent obtenir un prêt pour construire une minilaiterie, ou encore des ONG peuvent la financer (36).
A ce stade, le nombre de fournisseurs de lait ne peut augmenter que dans la mesure où de nouveaux marchés sont atteints. Ces premières laiteries ne peuvent donc se développer que dans les villes traversées par une voie de communication, même précaire, donnant accès à d'autres villes, voire à une autre région. Dans le sud du Pará, des entrepreneurs ont choisi de produire du fromage de type mussarela, facile à produire et à transporter en camionnette bâchée vers les villes de l'intérieur du Nordeste semi-aride, où le fromage est rare et cher. Grâce à cette option, le volume de lait collecté a dépassé celui de la consommation urbaine locale et le bassin laitier a commencé à s'étendre.

Saisissant cette opportunité, des propriétaires de pick-up se lancent alors dans la collecte de lait. Rémunérés au prorata des quantités collectées, ils parcourent les pistes pour convaincre les producteurs de tirer le lait de leurs vaches. Ceux-ci y trouvent un double avantage : un revenu monétaire régulier, mais aussi la fin de leur isolement. En effet, pour fidéliser ses fournisseurs, le transporteur assure un service de transport pour les marchandises et les personnes (intrants, écoliers...). Chaque transporteur construit ainsi sa propre ligne de collecte, dont certaines atteignent $80 \mathrm{~km}$ de longueur. Elles constituent un capital précieux, dont les droits d'exploitation sont enregistrés en mairie.

L'espace pionnier, jusque là très hermétique à toute forme de transport ou d'échange, devient plus ouvert et mieux desservi pour la circulation des produits et des personnes. C'est le début d'une série de transformations de l'espace sous l'effet du développement d'une filière laitière qui s'organise rapidement. Le bassin laitier prend forme. Une fois cette phase atteinte, les changements 
s'accélèrent car des intérêts se combinent pour stimuler la production et la vente de lait aux industries naissantes.

\section{Multiplication des petites laiteries}

Les installations de petites laiteries se multiplient. Le procédé de transformation du lait le plus fréquent est la fabrication de fromages mussarela, facile à mettre en œuvre même avec des laits acides, et qui permet une conservation longue et à moindre coût du produit transformé. Le modèle décrit dans la configuration précédente est compétitif et fait des émules. Des petits réseaux peuvent apparaître sous l'action d'un entrepreneur investissant dans plusieurs communes voisines, voire sur plusieurs fronts pionniers. Des coopératives peuvent également voir le jour, comme à Tucuruí, mais elles restent relativement rares, l'action collective en général étant difficile à coordonner sur les fronts pionniers $(30,31)$.

Aucune concurrence, ni en amont ni en aval, ne vient freiner le développement de ces unités. Elles transforment relativement peu de lait, que ce soit au regard du potentiel de production sur les fronts ou de la taille des marchés consommateurs distants qu'elles atteignent. Les flux (quelques milliers de litres par jour et par laiterie) restent donc modestes vis-à-vis des capacités de production régionale. Mais chez les petits éleveurs de la zone, l'idée de produire du lait se répand ; la vente quotidienne de lait fournit aux familles un revenu d'appoint complémentaire de la vente périodique des veaux mâles. Une telle opportunité est d'autant plus précieuse qu'elle est rare : sur les fronts pionniers, les productions végétales sont difficiles à conserver, transporter et vendre, surtout quand l'exploitation agricole est située loin de la ville. La contractualisation entre l'éleveur et la laiterie reste sommaire : il n'existe aucun engagement à moyen terme de part et d'autre. Le paiement régulier du lait n'est pas pleinement garanti à ce stade.

Malgré cette incertitude, le choix de commercialiser le lait peut entraîner des changements dans le système de production, notamment en ce qui concerne la gestion des pâturages et de l'alimentation. Dans cet environnement amazonien que tous les pionniers découvrent, de nouveaux systèmes techniques sont ainsi créés par les éleveurs (14). L'accès aux services, facilité par les laiteries, joue un rôle considérable dans cette transformation, par exemple sur le plan sanitaire (35).

\section{Arrivée d'investisseurs, rachats}

La situation évolue avec l'arrivée de nouveaux investisseurs sur le front pionnier amazonien. Cette nouvelle configuration coïncide généralement avec le goudronnage de la route d'accès au front pionnier et la connexion au réseau électrique national.

Ces investisseurs cherchent en Amazonie les moyens de combattre la concentration horizontale qui sévit dans la filière nationale. Les faillites ou les rachats sont nombreux et pour les éviter il est essentiel d'augmenter le chiffre d'affaires. Une façon satisfaisante d'y parvenir est de capter plus de matières premières en s'emparant de nouveaux bassins de production.

En résumé, ces investisseurs cherchent à s'emparer d'un bassin, à se l'approprier. Ils visent la mise en place d'un réseau de laiteries qui couvre tout un territoire, sans concurrents. Leur force réside, d'une part, dans leur accès facile aux principaux marchés consommateurs du pays et, d'autre part, dans leur grande capacité d'investissement. Il s'agit le plus souvent de grosses structures de transformation et de distribution, fournissant les réseaux de supermarchés comme Carrefour, Pão de Açúcar... Ils ont de solides capacités financières pour offrir un prix plus élevé au producteur que le concurrent local ne peut le faire. Celui-ci perd alors ses fournisseurs ou, s'il essaie de suivre la hausse du prix, épuise sa trésorerie jusqu'à l'asphyxie. D'une façon ou d'une autre, il est contraint à terme d'accepter les propositions de rachat de sa laiterie et de sa clientèle. L'implantation des investisseurs suit ainsi les règles du capitalisme le plus libéral, comme c'est souvent le cas sur les fronts pionniers. Les quelques tentatives de coopératives se sont heurtées à un manque de trésorerie, de capacité d'investissement, et de réseaux commerciaux vers l'aval.

Enfin, outre la compétitivité par le prix, les industries cherchent à fidéliser les producteurs par une série de services. Cela se traduit par des accès facilités aux intrants et autres facteurs de production, par des services sur le plan de la famille (transport, parfois facilité d'accès à l'hôpital...), mais aussi par une pression sur les politiciens pour investir et aménager les zones de production. Les plus grosses laiteries peuvent réunir plus de votes et peuvent donc mieux marchander avec les candidats. En échange du vote des producteurs laitiers, le futur maire s'engage à donner la priorité à leurs secteurs pour l'électrification, l'aménagement et l'entretien des routes, les écoles et infirmeries rurales...

Sous l'action de ces investisseurs, les bassins laitiers s'étendent et se structurent rapidement. Ils entrent dans une nouvelle phase et le lait cesse d'être une production marginale dans l'économie locale. Il devient un véritable outil de développement régional (23).

\section{Développement d'un réseau de laiteries}

L'investisseur a déjà beaucoup avancé dans la constitution de son réseau, avec des unités drainant la production de tout le territoire et approvisionnant une unité centrale. Pour l'éleveur, aux effets positifs de la concurrence (prix et services dédiés élevés) s'ajoutent les effets positifs d'une concentration déjà avancée (pression sur les politiques, aménagements, transports, services publics). Par ailleurs, le grand nombre de producteurs recevant un revenu sûr et régulier permet l'émergence en zone rurale de magasins et services. Les petites vilas voient ainsi s'installer des pharmacies, des épiceries, des lojas agropecuária. Avec les écoles et infirmeries installées par la préfecture sous la pression de la laiterie, et l'entretien de la route assurée par les transporteurs de lait*, les hameaux situés au cœur des bassins laitiers connaissent une dynamique de développement qui déclenche des cercles vertueux (plus de producteurs acceptent de traire et vendre le lait, d'autres sont attirés dans la région...). Le lait devient le vecteur du développement régional (22).

Une des caractéristiques de ce modèle de développement est le maillage rapide de l'espace. Pour les investisseurs, peu importe la rentabilité immédiate de l'investissement, il s'agit d'occuper le terrain. Il y a donc un dialogue permanent entre le directeur du réseau, les transporteurs et les responsables de chaque unité, pour savoir où implanter les prochaines unités. L'implantation d'une laiterie a de tels effets d'entraînement, qu'elle devient très rapidement rentable. Une nouvelle laiterie peut en quelques semaines atteindre un volume de 1000 ou 2000 L/jour, ce qui est suffisant pour rentabiliser les installations.

A ce stade, la totalité du lait cru est transformée dans ces unités qui possèdent donc chacune un bassin d'approvisionnement indépendant. Les produits fabriqués sont uniquement du fromage, surtout du mussarela, parfois du provolone ou du parmesan. Grâce à un volume de production croissant, la firme peut investir dans de nouveaux équipements pour diversifier la production et augmenter la valeur ajoutée : beurre, yaourts et lait pasteurisé. Il faut pour cela acquérir les équipements adéquats, construire de nouveaux bâtiments ou agrandir ceux qui existent. C'est le premier pas d'une réforme du réseau, qui aboutit à la phase 6 .

* Le transport de lait est sous-traité par la laiterie à des particuliers ou des firmes. 


\section{Spécialisation du bassin - monopole}

Toutes les fonctions de transformation du lait sont concentrées dans une unité centrale, dotée d'une capacité de plusieurs centaines de milliers de litres par jour et construite en fonction des normes sanitaires en vigueur au niveau international. Les unités réparties sur l'ensemble du bassin de production sont remplacées par des postes de réfrigération, vidés tous les deux ou trois jours par une flotte de camions-citernes. Acheminé à l'unité centrale, le lait est transformé en de nombreux produits, variables selon les époques de l'année et les configurations du marché.

Le transport réfrigéré du lait cru permet des gains significatifs sur les coûts de transport de la matière première, que les auteurs ont évalué à environ 0,1 US\$ par litre, soit environ 10 p. $100 \mathrm{du}$ prix payé au producteur. Cette économie peut être répercutée sur le prix au producteur, ce qui permet d'éradiquer définitivement les petites entreprises concurrentes dont la collecte est encore assurée par des transporteurs indépendants en camionnettes. Commencent alors de nouvelles évolutions, que l'Amazonie orientale ne connaît pas encore, mais qui s'observent depuis longtemps dans la région voisine du Goiás et plus récemment dans le Rondônia (Amazonie occidentale).

Fort de son monopole sur le bassin, le réseau n'a plus les mêmes contraintes vis-à-vis des producteurs. Le prix au producteur est remis en question. C'est le début du mode de rémunération en fonction de la qualité du lait ou de l'imposition de quotas distinguant basse et haute saison. Dans l'un comme dans l'autre cas, la tarification incite fortement le producteur à faire évoluer son système de production vers une plus grande productivité et une spécialisation laitière. Ainsi la vente du veau, qui assure l'essentiel des revenus de l'élevage aux familles sur les fronts pionniers, devient secondaire. L'amélioration génétique en direction de races laitières, la complémentation alimentaire, le haut niveau d'intrants, l'utilisation d'une main d'œuvre spécialisée s'imposent et s'accompagnent d'endettements des ménages de producteurs. La dépendance vis-àvis de la laiterie et de la rémunération qu'elle offre va en s'accroissant.

Une septième phase devient alors probable, avec le rachat du réseau par une multinationale. Les géants du secteur ne veulent pas de toute façon assumer les premières étapes du processus, décrites ci-dessus. Le bassin ne les intéresse qu'une fois structuré, contrôlé sur le plan des volumes de production, avec une qualité normalisée de la matière première.

\section{- EVOLUTION DE BASSINS LAITIERS AU MALI}

\section{Un potentiel laitier émerge de l'intégration agriculture - élevage}

$\mathrm{Au}$ Mali comme dans toute la zone sahélienne, la production laitière a longtemps été l'apanage des sociétés de pasteurs nomades, notamment peuls, qui vivent essentiellement de l'exploitation de leurs troupeaux (bovins et petits ruminants). Le lait a ici un rôle social, nutritionnel, économique, culturel, de premier plan (6). Il n'existe pas d'équivalent en Amazonie. Depuis les années 1970, l'élevage bovin s'est sensiblement déplacé vers le sud ; les zones soudaniennes et soudano-guinéennes sont devenues des bassins d'élevage bovin de premier plan (32). Le recul des barrières sanitaires au sud (trypanosomoses et onchocercose principalement) et les graves sécheresses au Sahel $(1973,1984)$ ont amorcé cette descente de l'élevage vers le sud. Le changement des systèmes agraires l'a accélérée et surtout pérennisée. En effet, l'introduction à partir des années 1950-1960 de la traction animale puis du « paquet technique » lié à la culture du cotonnier ont poussé les cultivateurs de la zone à acquérir des premiers bœufs de labour, puis à épargner leurs revenus cotonniers dans l'élevage bovin. A partir des années 1970, le développement de systèmes de culture en champs permanents plutôt qu'en rotation avec des jachères a rendu essentielle pour l'agriculture la disposition de fumures organiques, motivant encore un peu plus le développement de noyaux d'élevage bovin dans les exploitations. Cette intégration entre l'agriculture et l'élevage s'est fortement développée dans les années 1980 et 1990. Elle a abouti à la situation agraire actuelle, marquée par un potentiel élevé en productions animales, pour la viande et le lait, mais que les filières actuelles de commercialisation ont du mal à exploiter. Une dynamique comparable s'est développée en zone irriguée (zone Office du Niger), à partir de la culture du riz et de l'élevage transhumant.

En dépit d'un réel potentiel de production, les bassins laitiers émergent difficilement. La consommation rurale est faible ; elle n'oriente guère vers une exploitation laitière du troupeau, si ce n'est pour l'autoconsommation familiale, parfois le salaire du berger, voire l'obtention de revenus très ponctuels (2). Poccard-Chapuis et coll. (28) montrent ainsi que si 90 p. 100 des exploitations de la zone cotonnière, au sud du Mali, possèdent des bovins, moins de 10 p. 100 déclarent vendre du lait. Près des villes secondaires, des systèmes de vente directe existent, mais les volumes restent modestes et les élevages ne se spécialisent pas ou peu (20). A proximité de la capitale, la valorisation foncière interdit les systèmes extensifs et seuls se développent quelques élevages intensifiés, fondés sur le métissage d'animaux laitiers (Montbéliardes notamment), propriétés de riches fonctionnaires ou commerçants.

Des petites laiteries apparaissent et se développent (7), mais leur croissance reste souvent entravée par des difficultés de transport, par la petite taille des marchés consommateurs proches et par la distance du marché consommateur de la capitale, seul capable d'absorber des volumes conséquents (cf. ci-dessous). Une étude conduite entre 2004 et 2006 sur la consommation de produits laitiers à Bamako, Sikasso, Ségou et Mopti montre pourtant que le lait local n'est pas proposé au consommateur à un prix structurellement supérieur aux laits importés (27). La compétitivité basée seulement sur le prix des importations n'est donc pas un argument suffisant pour expliquer ce blocage, surtout dans le contexte de l'évolution récente du marché mondial du lait.

Le potentiel de production et les marchés consommateurs existent, mais les flux entre les deux restent très faibles, au contraire du cas amazonien. C'est donc bien la question de l'organisation du bassin laitier et de son fonctionnement qui se pose.

\section{Application au Mali du modèle amazonien}

\section{Un territoire aussi contraignant}

Dans les deux régions étudiées, les contraintes liées au territoire sont assez similaires. La production laitière est le fait de systèmes d'élevage relativement neufs ou en construction (fronts pionniers d'un côté, systèmes périurbains ou d'intégration agriculture - élevage de l'autre). Des deux côtés, les productivités sont faibles et le lait n'est qu'un sous-produit d'une activité d'élevage tournée vers la viande ou l'épargne, ou encore la traction animale au Mali. L'intensification de l'alimentation, la prophylaxie sanitaire, la modernisation des installations rurales, l'amélioration génétique sont peu ou ne sont pas mises en œuvre. La vente de lait ne procure qu'un revenu d'appoint, intéressant par sa régularité et son aptitude à prendre en charge des dépenses domestiques ou courantes. Rares sont les investissements visant à développer spécifiquement l'activité laitière, à l'exception de part et d'autre de quelques élevages périurbains appartenant à des fonctionnaires ou des commerçants. 
Dans les deux régions, la production est très atomisée, la circulation routière difficile et la collecte de lait cru problématique. Le coût de l'énergie et la difficulté à acquérir des équipements performants freinent les initiatives pour développer une industrie de transformation du lait cru ou la mise en place d'une chaîne du froid. Le pouvoir d'achat des consommateurs locaux ne permet guère de marges bénéficiaires dans la filière. Dans un cas comme dans l'autre, les territoires concernés sont très peu dotés en infrastructures et en services, les circuits commerciaux en amont et en aval sont peu nombreux. Les marchés locaux sont insuffisants pour permettre un développement significatif de la filière et la structuration d'un véritable bassin laitier. Les gros centres de consommation sont éloignés, difficiles d'accès et bien approvisionnés par des filières internationales.

\section{Configuration initiale similaire au modèle amazonien}

La première configuration du modèle de développement laitier élaboré sur le cas amazonien est fréquente sur l'ensemble de la zone rurale soudano-sahélienne. Elle se caractérise par l'absence d'unités de transformation. La vente de lait a lieu directement du producteur au consommateur, ou par l'intermédiaire d'un transporteur à bicyclette ou à moto. Ainsi, dans les villages et autour des chefs-lieux de la zone cotonnière du Mali, quelques exploitations vendent du lait aux villageois pendant la saison favorable à la production. La plupart des familles possèdent des bovins et n'achètent pas de lait, ou très peu (28).

Comme en Amazonie, on peut parler de bassins laitiers embryonnaires, de petite taille, très peu spécialisés, déconnectés entre eux. Les facteurs de compétitivité qui justifient cette organisation des bassins laitiers sont les mêmes : faibles niveaux de consommation, dispersion des éleveurs, meilleure rémunération des producteurs par les circuits de vente directe que par la transformation en laiteries. De plus, dans ces zones périurbaines il est souvent plus rentable de se consacrer à d'autres activités agricoles qu'à la production de lait : maraîchages et tubercules disposent de puissantes filières périurbaines, comme l'indiquent Bellinguez (1) et Le Masson (16). L'élevage périurbain pose au contraire des problèmes, notamment de mobilité des troupeaux dans un environnement occupés par les cultures.

\section{Emergence de petites unités de transformation, comme en Amazonie...}

La configuration suivante du modèle amazonien s'applique bien au Mali. Depuis une dizaine d'années, des minilaiteries s'installent près des villes secondaires de 30000 habitants ou plus, pour transformer le lait cru local et vendre des produits transformés sur le marché urbain. Le réseau «Danaya Nono » a été pionnier en ce domaine, avec des implantations réussies à Koutiala, Niono, San, dès le milieu des années 1990 (4). D'autres implantations ont été des échecs, comme à Sikasso ou à Ségou $(5,3)$. Ce réseau est né de l'initiative d'une ONG internationale, qui a vu dans le modèle des minilaiteries un moyen de lutter efficacement contre la pauvreté rurale et d'améliorer la nutrition dans les villes maliennes. L'ONG a assuré dans un premier temps les investissements dans les structures et les équipements, la formation des ressources humaines, la gestion du réseau. Progressivement ces fonctions ont été assumées par les laiteries elles-mêmes et l'ONG s'est retirée. L'achat du lait cru fait l'objet d'une contractualisation formelle entre la laiterie et les éleveurs avec des engagements réciproques sur les prix, les quantités et les services. Le modèle fonctionne d'autant mieux que les laiteries fournissent ces services d'appui à ceux-ci. L'accès aux produits vétérinaires et surtout aux compléments alimentaires (tourteau de coton, son de riz) étant problématique au Mali, les éleveurs trouvent là un motif supplémentaire d'adhésion à la laiterie. Plus récemment, les producteurs ont commencé à être partie prenante du capital des laiteries. Ce modèle organisationnel des minilaiteries est donc similaire aux configurations 2 et 3 des fronts pionniers amazoniens.

Les minilaiteries Danaya Nono font ainsi des émules. Dans la périphérie de Bamako, d'autres ONG (Vétérinaires sans frontières à Koumantou, à Kasséla) ou des organismes de coopération internationale (coopération française à Keleya, Ouelessebougou) se sont lancées dans cette activité.

\section{...mais la montée en puissance n'a pas lieu}

Pourtant, les configurations suivantes du modèle amazonien n'ont pas lieu au Mali. Les bassins laitiers maliens restent à un stade initial, peu efficace en termes de développement. En effet, ce n'est que dans la quatrième configuration du modèle que la filière laitière assure pleinement une fonction d'organisation de l'espace. Elle le rend plus performant pour son propre fonctionnement, elle façonne son territoire en un « outil de production » efficace, selon l'expression de Brunet et coll. (3). Deux mécanismes essentiels à cette évolution n'ont pas cours au Mali : le transport des produits finis vers des marchés distants et la collecte de lait frais dans un rayon supérieur à celui de la vente directe. Concernant le premier, l'évolution du bassin est liée à un flux essentiel : l'expédition des produits transformés au-delà du marché local (flèche rouge dans les modèles 2 et suivants). En l'absence de ce débouché, les flux sont trop faibles pour déclencher les mécanismes de structuration du bassin laitier. Dans un tel contexte, des mécanismes inverses, déstructurant, peuvent se mettre en place.

Malgré un marché saturé, de nouvelles minilaiteries peuvent voir le jour ; par imitation d'un système qui initialement a fonctionné, des entrepreneurs locaux (souvent des femmes) se lancent dans la production de yaourts ou de lait caillé par exemple (10). C'est tout un secteur informel qui se développe autour de la transformation de lait. Dans ce contexte concurrentiel, le prix au consommateur chute et les marges en amont se réduisent. Du coup les conditions offertes aux producteurs plafonnent ou régressent (5). La production en est d'autant plus atomisée, les volumes traits dans chaque exploitation en sont d'autant plus faibles et les coûts de collecte d'autant plus élevés (12).

Le lait cru cesse alors d'être une matière première compétitive, y compris dans le bassin laitier. Dans ces territoires peu aménagés, un entrepreneur installé en ville peut avoir plus de facilité à acquérir des sacs de poudre importée depuis la capitale, que du lait cru acheminé depuis les fermes environnantes. Une telle tendance affecte la plupart des transformateurs de produits laitiers au Mali, quelle que soit leur taille (21). Seuls les établissements se dotant d'une vocation militante pour le développement rural refusent cette stratégie, pourtant plus avantageuse sur le plan logistique. Ainsi la flèche rouge qui permet le passage vers la quatrième configuration non seulement ne se met pas en place, mais cède la place à un mouvement inverse. Le marché local consommateur naissant s'approvisionne hors du bassin de collecte, sur le marché mondial. Le bassin laitier reste bloqué à un niveau embryonnaire, tandis que se développe une concurrence accrue en aval, mais autour de produits dérivés de la poudre de lait. Le transformateur, informel, n'a pas de difficulté à tromper le client sur l'origine du produit aux moyens d'étiquettes annonçant un produit local.

Dès lors, le bassin laitier ne peut évoluer positivement que si la laiterie réussit à établir un flux d'expédition vers un marché distant. Elle pourra alors augmenter ses volumes et retrouver une influence positive sur les producteurs et leur territoire.

Le deuxième mécanisme entravant l'évolution des bassins laitiers au Mali concerne la collecte de lait frais dans un rayon supérieur à celui de la vente directe. Les exigences des laiteries 
vis-à-vis des éleveurs (critères de prix, qualité du lait, régularité d'approvisionnement) constituent des contraintes sérieuses pour les éleveurs par rapport à un système de vente directe. Or, dans les couronnes périurbaines, l'existence d'un dense réseau de collecteurs à bicyclette offre aux éleveurs la possibilité de vendre le lait à un bon prix, sans engagement sur des volumes constants, sans critères légaux de qualité sanitaire et physico-chimique. Le système de vente directe valorise mieux les produits issus des systèmes extensifs qui prévalent dans la zone (16). La laiterie doit en effet amputer le prix au producteur pour couvrir ses propres coûts de fonctionnement. Elle doit aussi imposer des critères de qualité, non seulement au regard de la règlementation sanitaire en vigueur (les circuits dits informels de vente directe échappent complètement à la règlementation sanitaire), mais aussi parce qu'un lait cru de mauvaise qualité se transformera mal en produits dérivés, limitant d'autant les marges bénéficiaires de la laiterie (5). Enfin, la laiterie doit fonctionner avec un volume de matières premières constant, équilibré en fonction du marché. Pour un éleveur situé près de la ville, vendre son lait à la laiterie n'est donc pas le meilleur calcul. A moins que celle-ci puisse lui proposer des services suffisamment intéressants, en plus du prix du lait, comme c'est le cas autour de Koutiala avec la distribution d'aliments concentrés.

La laiterie ne peut donc se forger un bassin d'approvisionnement que dans un rayon plus large. La capacité de transport dont la laiterie se dote est donc l'élément clé pour la mise en place de la quatrième configuration du modèle. Ce transport concerne aussi bien l'amont, pour la collecte dans des zones appropriées, que l'aval pour la vente et la distribution des produits sur des marchés distants et porteurs.

\section{Configuration classique au Mali : un bassin laitier périurbain statique}

La plupart des laiteries maliennes sont installées dans les couronnes périurbaines de villes secondaires. Elles cumulent ainsi les deux freins au passage en configuration 4 : elles ne parviennent pas à expédier leurs produits vers le marché distant de Bamako ; elles s'approvisionnent dans la couronne périurbaine, là où les éleveurs ont le moins intérêt à les fournir de façon satisfaisante.

Ces laiteries sont donc bloquées en amont par une concurrence avec les filières de vente directe, et en aval par un marché trop étroit et rendu concurrentiel. Un tel modèle périurbain n'offre finalement que des possibilités très limitées de lutte contre la pauvreté et d'amélioration de la nutrition.

Depuis quelques années, sous l'impulsion de coopérations internationales, de nouvelles laiteries s'implantent avec une localisation plus pertinente : suffisamment proches de Bamako pour y expédier leurs produits, et suffisamment éloignées de la couronne périurbaine pour intéresser et fidéliser les éleveurs. Le modèle suggère que ces laiteries (Kasséla, Ouelessebougou, Keleya) ont plus de chances d'atteindre la viabilité économique et de générer du développement local au sein d'un bassin laitier dynamique, plus étendu et surtout plus spécialisé et structuré qu'il ne l'est actuellement.

\section{ENSEIGNEMENTS DU MODELE POUR LE MALI}

L'analyse comparative montre que les bassins laitiers maliens restent bloqués dans leur évolution au stade 2 ou 3 pour deux raisons principales : l'accès aux marchés distants et le rayon de collecte du lait cru. La question discutée ici est de savoir comment résoudre ces deux problèmes.

\section{Utilité du modèle de von Thünen}

L'agronome allemand Heinrich von Thünen a publié en 1826 un modèle expliquant la localisation des activités agricoles, qui a marqué jusqu'à aujourd'hui les théories de l'économie spatiale (15). Dans un environnement théorique de «l'Etat isolé », où l'auteur fait abstraction des contraintes de relief, réseaux de transports, multiplicité des marchés consommateurs, l'analyse économétrique aboutit à la délimitation de six couronnes concentriques, disposées autour du marché consommateur urbain. Plus on s'éloigne du centre, plus le coût d'accès à la terre décroît alors que le coût du transport s'élève. Ainsi, chaque activité agricole trouve sa place dans l'une ou l'autre des couronnes en fonction, d'une part, de la valeur ajoutée qu'elle permet de produire par unité de surface, d'autre part, des coûts de transport qui affectent ses produits. Dans le cas du lait, von Thünen distingue deux localisations possibles, suivant le type de produit (34). Les produits frais sont localisés dans la première couronne, un trop long transport n'est pas compatible avec la périssabilité du produit dans le contexte du XIX ${ }^{\mathrm{e}}$ siècle (pas de motorisation, pas de chaîne du froid). A l'inverse, la production fromagère (et de beurre salé) est localisée dans la dernière couronne : le produit se conserve facilement et sa forte valeur économique par unité de poids fait que le coût du transport est relativement faible (par rapport à celui du gain). Il est donc plus intéressant de le produire là où la terre est la moins chère.

L'application de ce modèle au cas malien n'est pas dénuée d'intérêt. On observe bien dans la configuration classique de ce pays, que les bassins laitiers restent cantonnés à la couronne périphérique des villes pour produire du lait frais ou éventuellement du lait caillé. La question de la conservation et du coût du transport délimite l'enveloppe externe des bassins laitiers ; la limite interne n'existe pas, puisque la production a lieu également à l'intérieur de la ville. Toutefois, les déterminants de la localisation des productions se sont complexifiés, ce qui a poussé les auteurs à chercher une autre échelle d'analyse, celle du bassin laitier.

En effet, von Thünen justifie la présence du lait dans la première couronne, malgré sa forte emprise sur le foncier, par le fait que le fumier y est essentiel pour maintenir la fertilité des sols consacrés au maraîchage et par l'existence d'un marché pour les produits frais ; la question de la fumure, mais aussi du travail animal, joue aussi pour les cercles 2 à 5 . La vache ne sert à produire que du lait ; elle transforme aussi de la matière végétale (fourrages et litières) en fertilisants, difficiles à transporter. Cette double fonctionnalité, ainsi que la proximité d'un marché pour les produits frais et le travail animal pour les cultures justifient la présence de l'élevage laitier dans la première couronne. Au Mali, cette logique perdure, les fumiers sont précieux près de la ville, pour produire des légumes. Mais les engrais chimiques ont mis un point final au caractère incontournable de la présence de bovins dans ces zones de culture intensive. Dès lors, maintenir des bovins à proximité de la ville peu poser des problèmes, comme à Sikasso où l'espace agricole, occupé par un maraîchage, est très développé dans la première couronne (8). On assiste au même processus à Bamako avec la conversion rapide des terrains agricoles en terrains constructibles.

D'autre part, les techniques de transport et de conservation ont évolué, avec au Mali le transport routier, la conservation par le froid (réfrigérateurs ou glacières), voire par la lactoperoxydase. Les techniques de transformation industrielle du lait cru se sont développées, alors qu'à l'époque de von Thünen la transformation était artisanale. Du coup, moyennant l'installation sur place d'unités de transformation, il est devenu possible de délocaliser la production vers des zones plus éloignées, sans nécessairement 
aller jusqu'aux zones les plus périphériques. Par ailleurs, l'élevage étant aussi une source d'énergie pour les travaux des champs (traction animale), le potentiel de production laitier se trouve également dans des zones qui, dans le modèle de von Thünen, sont plus propices aux cultures céréalières (mais pas au maraîchage, puisqu'au Mali le travail du sol y est manuel).

En d'autres termes, les principes qui soutiennent le modèle de von Thünen sont toujours valables, y compris au Mali dans le cas du lait. Mais ils souffrent de plusieurs distorsions qui compliquent sa lecture à tel point que le modèle perd de son opérationnalité et de sa simplicité, qui faisaient sa force pour organiser les territoires agricoles. De ce fait, il a semblé plus pertinent de construire une autre représentation de la réalité et du fonctionnement des bassins laitiers. Bien qu'elle ne renie pas les fondements de von Thünen, la présente approche permet d'intégrer les localisations respectives des éleveurs, des transformateurs et du marché, tout en intégrant les paramètres actuels des contraintes de transport, de conservation et de transformation des produits. Cette meilleure prise en compte de la réalité permet de proposer des pistes pour mieux promouvoir l'expansion des bassins laitiers maliens.

\section{Assurer le principe de fluidité}

La maîtrise d'un approvisionnement régulier en quantité et en qualité, d'une part, et celle de la distribution de produits laitiers satisfaisants pour les consommateurs, d'autre part, représentent le souci majeur des opérateurs de collecte. Le contrôle des flux de produits laitiers sous-tend par conséquent le développement de l'activité (33).

\section{Choisir les lieux d'implantation de laiteries}

La laiterie est l'élément central qui peut favoriser l'évolution du bassin. Ce constat fondamental tient au fait qu'en l'absence d'unités de transformation, le bassin laitier est cantonné à la zone périurbaine à cause des transports, et est limité en volume du fait de l'intérêt limité à produire du lait dans cette zone. La ville consomme alors du lait en poudre. C'est la deuxième configuration du présent modèle : absence de réelles possibilités d'expansion du bassin laitier et d'impacts significatifs sur le développement local, a fortiori sur la sécurité alimentaire du pays. La question qui se pose alors est celle de la localisation de la laiterie pour s'affranchir au mieux des contraintes de transport aussi bien en amont qu'en aval.

En amont, l'approvisionnement doit pouvoir s'affranchir de la concurrence des lignes de collecte en vente directe. Une implantation en zone périurbaine n'a donc pas de sens de ce point de vue. S'implanter en zone rurale est la solution la plus efficace, mais la laiterie doit néanmoins être proche d'un axe routier goudronné et fréquenté pour acheminer les produits vers la capitale. L'électrification est aussi un critère important, mais pas essentiel (possibilité de groupe électrogène ou d'énergie solaire). La laiterie doit nécessairement organiser un système de collecte efficace et peu coûteux sur de longues distances. Cela peut passer par une flotte motorisée (motos, voitures) et par des centres de collecte villageois, qui réduisent le coût de transport. La lactoperoxydase pourrait ici être utile dans la mesure où elle permet de conserver plus longtemps le lait dans ces centres de collecte (19). Le résultat serait équivalent à celui de la mise en place des centres de collecte réfrigérés qui émergent en Amazonie (configuration finale du modèle). Un volet « appui aux éleveurs » est souhaitable pour fidéliser les éleveurs et ainsi contrôler les volumes de matière première, pour améliorer les productivités des élevages et ainsi augmenter les volumes collectés sans augmenter les coûts fixes de transport, et pour améliorer la qualité du lait cru pour une meilleure transformation du lait et qualité des produits finis.
En aval, l'objectif est d'accroître les volumes commercialisés, ce qui implique de prendre des parts sur des marchés distants. Se pose une première difficulté qui est celle du transport des produits finis vers ces marchés. En Amazonie, la transformation en fromage mussarela résout en bonne partie ce problème. Au Mali, ce type de produits est trop peu consommé, les produits frais dominent. La réfrigération est donc nécessaire. L'utilisation de camions frigorifiques est idéale, mais dans un premier temps des glacières, même artisanales, sont suffisantes. Elles peuvent être transportées par des véhicules appartenant à la laiterie ou par les bus qui desservent Bamako.

\section{Organiser la distribution à Bamako}

La deuxième difficulté est celle de la distribution des produits à Bamako. Les consommateurs s'approvisionnent essentiellement à proximité immédiate de leur domicile (27). Il n'existe pas ou peu de grands centres de distribution (tels que les supermarchés au Brésil). Il n'est donc pas envisageable d'approvisionner un distributeur qui se chargerait d'atteindre le consommateur. La laiterie doit assurer cette fonction et donc trouver un mode de livraison de ses produits adapté à une telle atomisation de la distribution. Il est donc essentiel de se doter d'un appareil efficace de distribution. La firme Nestlé, par exemple, dispose d'une flotte de motos et véhicules à trois roues qui sillonnent la ville, approvisionnant les distributeurs. Un modèle plus économique est celui des bicyclettes équipées de glacières, parcourant la ville et ses points de forte consommation, tels que les écoles, les établissements publics, les marchés... Ainsi une laiterie malienne qui viserait le marché de la capitale devrait se doter d'un centre de stockage des produits à Bamako (ou de plusieurs, dans différents quartiers), approvisionné régulièrement par les nouveaux arrivages de la laiterie. Une flotte de bicyclettes contractualisée peut alors assurer la distribution des produits.

Enfin la troisième difficulté relève du marketing. Elle tient à l'image du produit, qui doit être valorisée par rapport à celle des produits importés ou élaborés à partir de poudre importée. Sans cette valorisation de l'origine locale du produit et de la qualité nutritionnelle qui en découle, les atouts de la poudre restent décisifs : facilité de distribution (transport, stockage), image de qualité sanitaire irréprochable... Cette image des produits locaux devra aussi faire l'objet d'une réglementation vérifiant l'origine locale, pour éviter le piratage de l'image par des produits en réalité issus des importations (11).

\section{Difficultés spécifiques au Mali}

La consommation de produits laitiers au Mali est relativement différente de celle observée au Brésil, ce qui bien entendu n'est pas sans conséquences sur la filière et le bassin. Comme déjà mentionné, la présente démarche n'est pas de prétendre exporter au Mali les méthodes qui fonctionnent au Brésil. L'application du modèle doit contribuer à faire ressortir les spécificités des différents contextes. Certaines ne sont pas explicites dans le modèle mais ne doivent pas être oubliées pour autant (contexte culturel, systèmes économiques notamment).

\section{Les Maliens consomment peu de fromages}

La montée en puissance des laiteries en Amazonie repose sur un produit facile à produire et à transporter : le fromage mussarela. On peut dire que les premières configurations du modèle sont celles de bassins fromagers. Peu sensible aux variations de qualité du lait, d'un goût relativement uniforme, surtout si le fabricant ajoute beaucoup de sel, supportant un transport non réfrigéré de plusieurs heures, ce fromage est un produit idéal pour les territoires enclavés et les systèmes d'élevage très extensifs (35). De plus, il est 
très demandé sur les marchés urbains pour la consommation « bas de gamme », telle que celle des vendeurs de pizzas ou sandwichs dans les rues ou sur les plages. Un tel type de production n'est pas transférable au Mali, puisque le fromage y est très peu consommé. Même les récents changements de comportements des consommateurs ne laissent pas présager de croissance du marché du fromage. Il constitue un marché de niche pour les classes aux revenus les plus élevés et parfois une consommation ponctuelle pendant le ramadan. Ces changements concernent plutôt les yaourts ou laits caillés, sucrés et aromatisés (27).

Les laiteries maliennes n'ont ainsi pas d'autres options que d'élaborer des produits frais, tels que le lait pasteurisé, les laits caillés, les yaourts. Le cas du beurre clarifié n'est guère plus porteur que celui du fromage, dans la mesure ou sa production reste traditionnelle et la commercialisation totalement informelle. Ces produits ont l'avantage de ne pas demander d'affinage donc de chambres froides. En revanche, ils nécessitent une chaîne du froid en aval, donc une contrainte logistique et des coûts supplémentaires pour le transport et la distribution. L'utilisation de glacières semble la solution la plus viable, au moins dans un premier temps, le transport en camion frigorifique n'étant pas rentable pour de petits flux (19). Le fait que le Malien ne consomme pas de fromages est une difficulté supplémentaire, mais l'emploi de techniques simples pour conserver des produits frais par le froid pourrait la résoudre.

\section{Faible consommation urbaine}

A la différence de celles du Brésil, les industries agro-alimentaires maliennes ne peuvent pas encore compter sur un large marché intérieur pour assurer leur développement. Les niveaux de consommation per capita sont mal connus dans les villes du Mali, mais n'excèdent pas quelques dizaines de litres par an pour une majorité d'individus (2) La faiblesse des pouvoirs d'achat est un frein incontestable au développement de la consommation des produits laitiers. Par ailleurs, le faible taux d'urbanisation (taille et nombre des villes) limite beaucoup la demande en produits laitiers au Mali. Avec 70 p. 100 de population rurale, les villes représentent une demande comparativement très faible pour les produits agricoles. Cette réalité peut cependant évoluer très vite. Le mouvement d'exode rural est amorcé et l'un des enjeux des filières agricoles locales est de parvenir à profiter de la nouvelle demande alimentaire des villes ; le lait ne fait pas exception (9).

Enfin, la maille urbaine malienne est très «macrocéphale »: Bamako regroupe 43 p. 100 de la population urbaine du pays, et les villes secondaires sont de petite taille (entre 130000 habitants pour Sikasso et 40000 pour Kayes). Ainsi, les marchés des plus gros chefs-lieux amazoniens sont d'une taille équivalente à celle de la douzaine de villes secondaires que compte le Mali. Pour passer à la configuration 4, seul le marché de Bamako est capable d'absorber les volumes suffisants de produits laitiers. Les laiteries maliennes doivent indiscutablement capter ce marché, pour espérer grossir et avoir un impact significatif sur le développement rural. Les seuls bassins laitiers significatifs au Mali, en termes de développement local, seront ceux qui atteindront le marché bamakois. A ce jour, aucun ne s'est durablement profilé.

\section{Question du passage à l'ère industrielle}

A l'instar du Brésil et des pays occidentaux, l'industrialisation laitière permet de soutenir le développement de régions de production. Certes, cette industrialisation a ses limites, souvent évoquées dans les critiques altermondialistes. La multiplication des minilaiteries, d'une capacité de quelques centaines de litres par jour, préserve sans doute davantage l'environnement et les rapports sociaux. Mais, comme cela a déjà été souligné, leur impact national est quasi nul.
Mali-Lait et Eurolait possèdent aujourd'hui l'outil industriel, d'une capacité de $20000 \mathrm{~L} / \mathrm{j}$. Mais les usines sont situées au cœur de Bamako, ce qui les rend proches tout à la fois des sources d'approvisionnement (poudre de lait importée) et des débouchés solvables (consommation urbaine). Dans un contexte d'augmentation sensible du prix mondial de la poudre, l'offre locale devient néanmoins intéressante. Pour la collecte de lait cru, ces entreprises s'appuient sur quelques gros éleveurs intensifs et sur des coopératives de collecte basées en périphérie de Bamako. Pour autant, le principe de fluidité de l'approvisionnement n'est pas assuré aussi bien en termes de quantité (faiblesse de l'offre, espace en voie de saturation) que de qualité sanitaire. Les industriels demeurent par conséquent extrêmement prudents.

Au-delà de l'outil laiterie, il importe donc de considérer le problème de l'approvisionnement sous l'angle des rapports entre producteurs et transformateurs. De ce point de vue, les éleveurs brésiliens se démarquent clairement de leurs homologues maliens. Face à des esprits d'entrepreneurs amazoniens, les soudano-sahéliens renvoient souvent une image de sociétés communautaires. Si la situation n'est pas figée $(6,17)$, il n'en reste pas moins que la transition à la société capitaliste ne se fait pas sans heurts ni sans freins. Au demeurant, l'esprit coopératif est paradoxalement loin d'être répandu chez les pasteurs sahéliens. L'efficacité de la collecte passe pourtant par ce canal. En dehors des laiteries et des politiques locales et nationales, les producteurs laitiers ont aussi leur part de responsabilité dans les blocages de la filière. Le modèle brésilien montre que les deux évoluent ensemble vers une organisation plus efficace. Les éleveurs seuls ne peuvent assumer la charge d'organiser la collecte, puis la transformation et la distribution des produits.

\section{CONCLUSION}

La modélisation des dynamiques de développement des bassins laitiers en Amazonie est riche d'enseignement pour comprendre les blocages qui caractérisent la situation malienne. Elle montre qu'au Mali l'évolution des bassins reste bloquée à un stade sinon initial, du moins peu avancé. L'impact que pourrait avoir le lait sur le développement régional reste donc faible à l'échelle locale et insignifiant à l'échelle nationale.

L'application du modèle montre que les clés de l'évolution ne se situent pas au niveau des techniques de production. De nombreux travaux ont été faits dans la zone sur les systèmes de production laitière, leur intérêt économique pour la vente de lait, leurs effets positifs sur la production de fumure organique ou l'état sanitaire des troupeaux. Ces travaux n'ont guère permis à la production et aux filières laitières de prendre de l'essor.

La présente analyse montre au contraire que c'est au niveau des laiteries que doivent d'abord porter les mesures d'appui. Les fonctions de transport sont au cœur de la problématique. Von Thünen l'avait bien montré pour l'Europe du XIX ${ }^{\mathrm{e}}$ siècle. La réalité malienne d'aujourd'hui offre toutefois d'autres solutions qu'une localisation exclusive dans la première couronne, décrite dans le modèle de von Thünen, pour les produits frais et dans la dernière pour des fromages. La collecte, la transformation et la distribution des produits laitiers sont les fonctions essentielles qui permettent au bassin laitier de prendre de l'ampleur, puis de s'organiser de façon de plus en plus performante, gagnant en compétitivité et générant des effets positifs sur l'économie des exploitations agricoles, la qualité de vie des ruraux et le développement territorial. Des moments clés ponctuent ce processus d'organisation, qui font chacun appel à des initiatives ou des solutions spécifiques. Le modèle indique qu'au Mali les bassins laitiers périurbains actuels 
sont bloqués par ces questions de transport en amont et en aval de la laiterie.

Toutefois, une laiterie a besoin d'appuis pour développer ses fonctions où la logistique joue un rôle central. Elle doit disposer d'un capital d'investissement important, pour s'équiper (ou contractualiser des sous-traitants), notamment en moyens de transport (en amont en en aval). Elle doit aussi disposer de ressources humaines bien formées, capables d'assurer une intégration verticale entre des fonctions, donc des métiers, très différentes (de la collecte du lait cru à la distribution de produits transformés). Cette intégration suppose également une gestion fine et efficace. Enfin, le fonctionnement de cette laiterie n'est viable que dans un cadre réglementaire adéquat qui la protège notamment des concurrences déloyales (filières informelles, utilisation abusive de l'image de produit local). Cela suppose également une connaissance fine du marché consommateur.

L'Etat malien, ainsi que toutes les organisations qui souhaitent exploiter le potentiel laitier pour générer un développement régional, devraient réorienter leurs appuis en fonction de ces éléments. Ce n'est qu'en faisant sauter le premier verrou de l'accès aux marchés distants, puis celui de la collecte sur un large rayon, que la dynamique positive observée en Amazonie pourra se développer également au Mali.

\section{BIBLIOGRAPHIE}

1. BELLINGUEZ A., 1994. Etude de l'impact des projets sur les systèmes de production laitiers périurbains à Bamako. Mém. Ing. Tech. Agric. Rég. Chaudes. Montpellier, France, Eitarc/Cnearc, 98 p. + annexes.

2. BONFOH B., CORNIAUX C., COULIBALY D., DIABATE M., DIALLO A., FANE A., KONE Y., NAPO A., POCCARD-CHAPUIS R., TRAORE A., 2005. Synthèse bibliographique sur les filières laitières au Mali. Document de travail. Bamako, Mali, Réseau de recherches et d'échanges sur les politiques laitières, 76 p. http://www.repol.info/IMG/pdf/Synthese_ biblio_du_Mali.pdf

3. BRUNET R., FERRAS R., THERY H., 1998. Les mots de la géographie. Dictionnaire critique, $3^{\mathrm{e}}$ édn. Paris, France, Reclus / La documentation française.

4. CIDR, 1997. Organisation de la production, de la transformation et de la commercialisation des produits laitiers dans les villes secondaires du Mali. Rapport annuel $n^{\circ}$ 23, projet Cidr/Mali/Filière lait. Bamako, Mali, Cidr, 93 p.

5. CIDR, 1998. Organisation de la production transformation et commercialisation des produits laitiers. Rapport annuel $n^{\circ} 26$, projet Cidr/Mali/Filière lait. Bamako, Mali, Cidr, 28 p.

6. CORNIAUX C., 2005. Gestion technique et gestion sociale de la production laitière : les champs du possible pour une commercialisation durable du lait. Cas des systèmes d'élevage actuels du delta du fleuve Sénégal. Thèse Doct., INA-PG, Paris, France.

7. CORNIAUX C., DUTEURTRE G., DIEYE P.N., POCCARD-CHAPUIS R., 2005. Les minilaiteries comme modèle d'organisation des filières laitières en Afrique de l'Ouest : succès et limites. Revue Elev. Méd. vét. Pays trop., $58: 237-243$.

8. COULIBALY D., 2009. Commercialisation du lait : un moteur d'innovation dans les systèmes d'élevage en zone périurbaine de Sikasso au Mali. Thèse Doct., AgroParisTech, Paris, France.

9. DUFUMIER M., 2005. Etude des systèmes agraires et typologie des systèmes de production agricole dans la région cotonnière du Mali. Bamako, Mali, ministère de l'Agriculture, Programme d'amélioration des systèmes d'exploitation en zone cotonnière, 83 p.

10. DUTEURTRE G., 2000. Etude sur I'organisation des filières agro-alimentaires dans les pays de la zone de Solidarité prioritaire. L'organisation de la filière laitière autour de Niono (Mali). Rapport de mission. Paris, France, ministère français des Affaires étrangères, 20 p.
11. DUTEURTRE G., 2003. Normes exogènes et tradition locale : la problématique de la qualité dans les filières laitières africaines. In : Sémin. Lait sain pour le Sahel, Bamako, Mali, 25 avr. - 1 mars 2003. www.laitsain.ch.vu

12. FAO, 1996. Appui à la formation d'un programme national de promotion de la production laitière au Mali. Rome, Italie, FAO, 159 p. + annexes.

13. FAYE B., ALARY V., 2001. Les enjeux des productions animales dans les pays du Sud. Prod. Anim., $14:$ 3-13

14. HOSTIOU N., 2003. Pratiques et stratégies de gestion des ressources herbagères cultivées par des éleveurs laitiers sur un front pionnier en Amazonie brésilienne : le cas du municipe de Uruará. Thèse Doct., INAPG, Paris, France, 206 p.

15. HURIOT J.M., 1999. Von Thünen ; économie et espace. Paris, France, Economica.

16. LE MASSON A., 1996. Programme national de promotion de la production laitière au Mali. Les systèmes d'élevage à vocation laitière : contraintes, propositions. Montpellier, France, Cirad-emvt, 95 p. + annexes. (Rapport n 96-030)

17. LE NAY, VATIN F., 1993. L'industrialisation laitière impossible. In : Corbel J., Vatin F., éds, Mondes ruraux en mutation. Rennes, France, Presses universitaires.

18. MERTENS B., POCCARD-CHAPUIS R., PIKETTY M.G., LAQUES A.E., VENTURIERI A., 2002. Crossing spatial analyses and livestock economics to understand deforestation processes in the Brazilian Amazon: the case of Sao Félix do Xingu in South Para. Agric. Econ., 27: 269-294.

19. METZER R., CENTRES J.M., THOMAS L., LAMBERT J.C., 1995. L'approvisionnement des villes africaines en lait et produits laitiers. Rome, Italie, FAO, 102 p. (Prod. Santé Anim. n 124)

20. MORIN G., COULIBALY D., CORNIAUX C., POCCARD-CHAPUIS R., SIDIBE. S.I., MOULIN C.H., 2007. Dynamiques des unité de production laitière dans le bassin d'approvisionnement de la ville de Ségou au Mali. Revue Elev. Méd. vét. Pays trop., 60.

21. PINAUD S., 2007. Etude du réseau de distribution des produits laitiers à base de poudre de lait à Bamako. Mém. Master Sci. Hum. Soc., Université Paris 10, Nanterre, France, $100 \mathrm{p}$.

22. POCCARD-CHAPUIS R., BOUTONNET J.P., LAQUES A.E., PIKETTY M.G., TOURRAND J.F., 2001. Filières bovines et structuration des espaces pionniers en Amazonie orientale brésilienne. In : Coll. Un produit, une filière, un territoire, Toulouse, France, 21-23 mai 2001, $16 \mathrm{p}$.

23. POCCARD-CHAPUIS R., VEIGA J.B., PIKETTY M.G., MORELLY C.K.F., TOURRAND J.F., 2001. A cadeia produtiva do leite: uma alternativa para consolidar a agricultura familiar nas frentes pioneiras da Amazonia? Bol. Pesqui. EMBRAPA (Abril): $19 \mathrm{p}$

24. POCCARD CHAPUIS R., PIKETTY M.G., VEIGA J.B., TOURRAND J.F., 2003. Milk production, regional development and sustainability in the Eastern Brazilian Amazon. In : Coll. int. Les systèmes agroalimentaires localisés (Syal) : produits, entreprises et dynamiques locales, Montpellier, France, 16-18 oct. 2002.

25. POCCARD-CHAPUIS R., 2004. Les réseaux de la conquête. Rôle des filières bovines dans la structuration de l'espace sur les fronts pionniers d'Amazonie orientale brésilienne. Thèse Doct. Géogr., Université Paris $X$, Nanterre, France, 435 p. + annexes.

26. POCCARD-CHAPUIS R. THALES M.C, VENTURIERI A, PIKETTY M.G., MERTENS B., BASTOS DA VEIGA J., TOURRAND J.F., 2006. The livestock commodity chain: A lever for monitoring pioneer dynamics in the Brazilian Amazon Region? In: Changing European farming systems for a better future. New visions for rural areas. Wageningen, the Netherlands, Wageningen Academic Publishers, p. 430-434.

27. POCCARD-CHAPUIS R., CORNIAUX C., COULIBALY D., OUOLOGUEM B., 2007. La demande urbaine en produits laitiers : contrastes entre la capitale et les villes secondaires du Mali. In : 14 es Rencontres Recherches Ruminants, Paris, France, 5-7 déc. 2007.

28. POCCARD-CHAPUIS R., COULIBALY D., BA A., SISSOKO S., BENGALY M., 2007. Analyse affinée des pratiques et des stratégies paysannes. Rapport annuel du projet Caractérisation des dynamiques agraires dans les zones cotonnières du Mali. Bamako, Mali, ministère de I'Agriculture - Programme d'amélioration des systèmes d'exploitation en zone cotonnière, $199 \mathrm{p}$. 
29. ROSTOW W., 1963. Les étapes de la croissance économique. Paris, France, Seuil, 252 p.

30. TOURRAND J.F., GRIJALVA J., VALENCIA F., RIOS ALVAREDO J., POCCARD-CHAPUIS R., PIKETTY M.G., VEIGA J.B., 2001. Enjeu économique et défi écologique de la production laitière en Amazonie. In : $10^{\mathrm{e}}$ Conf. int. de I'Association des institutions de médecine vétérinaire tropicale, Copenhague, Danemark, 20-24 août 2001.

31. TOURRAND J.F., VEIGA J.B., Eds, 2003. Viabilidade de sistemas agropécuários na agricultura familiar da Amazônia. Belém, PA, Brasil, Embrapa Amazônia Oriental, 468 p.

32. VALL E., DUGUE P., BLANCHARD M., 2006. Le tissage des relations agriculture - élevage au fil du coton. Cah. Agric., 15 : 72-79.

\section{Summary}

Poccard-Chapuis R., Corniaux C., Coulibaly D. Structuring of Milk Production Basins: Comparison between Brazilian Amazonia and Mali

In Brazilian Amazonia, structuring of dairy basins is progressing fast. In Mali, these processes are not engaged, although the activity of cattle keeping is ancient and currently thriving. This difference covers essential stakes: fight against poverty, food production in the face of the demographic challenge, current debates on the theories of the development. The comparative analysis is based on a diachronic model of the successive phases of structuring of the Amazonian dairy basins. This model is then applied to dairy dynamics in Mali to identify blockages in the structuring of basins. Six phases are highlighted in the Amazonian model, from subsistence consumption of raw milk to basin specialization and establishment of an industrial monopoly. The application in Mali shows that the initial situations are similar (territory constraints). But two essential mechanisms are not working: transportation of finished products to distant markets, and fresh milk collection extended beyond the boundaries of the direct sale to urban consumers. These two aspects, essential to ensure the flow upstream and downstream of the products, address the question of dairy unit location as well as the distribution organization in Bamako. In spite of the limits of the comparative analysis, the model shows that the keys to a positive evolution in Mali are more to be found at the industry and distribution levels than at the production level. Public actions (State and collectivities) could focus on these two points, notably through training activities and credit policies.

Keywords: Dairy cattle - Agricultural development - Model Informal sector - Amazonia - Brazil - Mali.
33. VATIN F., 1990. L'industrie du lait, essai d'histoire économique. Paris, France, I'Harmattan, 223 p.

34. VATIN F., 1996. Le lait et la raison marchande. Essai de sociologie économique. Rennes, France, Presses universitaires de France, 205 p.

35. VIEIRA L.C., POCCARD-CHAPUIS R., VEIGA J.B., 2001. Produção e avaliação da qualidade do leite na microrregião de Castanhal. Belém, PA, Brasil, Embrapa Amazônia Oriental, 26 p. (Documentos n 95)

36. VEIGA J.B., TOURRAND J.F., Eds, 2000. Produção leiteira na Amazônia oriental. Situação atual e perspectivas. Belém, PA, Brasil, Embrapa Amazônia Oriental, 234 p.

Accepté le 28.08.2009

\section{Resumen}

Poccard-Chapuis R., Corniaux C., Coulibaly D. Dinámicas de estructuración de las mesetas lecheras: comparación entre el Amazonas brasileño y Malí

En el Amazonas brasileño, se llevan a cabo en este momento rápidos procesos de estructuración de las mesetas lecheras. En Malí, esta estructuración no a aún comenzado, aunque la cría bovina sea una actividad antigua y en plena expansión. Esta diferencia cubre dos cuestiones esenciales: la lucha contra la pobreza, producciones alimenticias frente al desafío demográfico, debates actuales sobre las teorías del desarrollo. El análisis comparativo se apoya sobre una modelización diacrónica de las fases sucesivas de estructuración de las mesetas lecheras amazónicas. Este modelo se aplica luego a las dinámicas lecheras de Malí para identificar los bloqueos de la estructuración de las mesetas. El modelo amazónico muestra seis fases, del autoconsumo de leche cruda hasta la especialización de la meseta y la instauración de un monopolio industrial. La aplicación en Malí muestra que las situaciones son similares (obstáculos ligados con el territorio). Pero dos mecanismos esenciales no funcionan: el transporte de productos acabados hacia los mercados distantes y la colecta de leche fresca en un radio superior al de la venta directa a los consumidores urbanos. Estos dos aspectos, esenciales para asegurar la fluidez anterior y posterior de la industria, conducen a revisar los lugares de implantación de las lecherías, así como la organización de la distribución en Bamako. A pesar de los límites del análisis comparativo, el modelo muestra que las Ilaves de una evolución positiva en Malí se sitúan más a nivel de las industrias y de la distribución que a nivel de la producción. Las acciones públicas (Estado y colectividades) podrían aportar hacia estos dos puntos, principalmente mediante la formación y las políticas de crédito.

Palabras clave: Ganado de leche - Desarollo agrícola Modelo - Sector informal - Amazonia - Brasil - Malí. 